\title{
1 Identification of Immunodominant T-lymphocyte Epitope Peptides \\ 2 in HPV 1, 2 and 3 L1 Protein for Novel Cutaneous Wart Vaccine
}

3

4 Wu Han Toh ${ }^{1,2}$, Chuang-Wei Wang ${ }^{2,4}$, Wen-Hung Chung ${ }^{2,3,4, *}$

$5 \quad{ }^{1}$ Department of Scientific Research, Taipei American School, Taiwan

$6{ }^{2}$ Cancer Vaccine and Immune Cell Therapy Core Laboratory, Chang Gung Memorial Hospital,

7 Chang Gung Immunology Consortium, Linkou, Taiwan

$8 \quad{ }^{3}$ College of Medicine, Chang Gung University, Taoyuan, Taiwan

$9{ }^{4}$ Genomic Medicine Core Laboratory, Chang Gung Memorial Hospital, Linkou, Taiwan

19 * Correspondence:

20 Wen-Hung Chung

21 chung1@.cgmh.org.tw

22

23

24

25 


\section{Abstract}

27 Background: Common warts and flat warts are caused by the human papillomavirus (HPV).

28 Peak incidence of wart infection occurs in schoolchildren aged 12-16, where prevalence can be as high as $20 \%$. Traditional treatments aimed at destruction of wart tissue have low clearance rates and high recurrence rates. Occasional reports have even shown warts becoming malignant and progressing into verrucous carcinoma. Current licensed HPV vaccines largely target higherrisk oncogenic HPV types, but do not provide coverage of low-risk types associated with warts. To date, little attention has been given to the development of effective, anti-viral wart treatments.

Objective: This study aims to identify immunodominant T-lymphocyte epitopes from the L1 major capsid protein of HPV 1, 2 and 3, a foundational step in bioengineering a peptide-based vaccine for warts.

Methods: Cytotoxic T-cell and helper T-cell epitopes were predicted using an array of immunoinformatic tools against a reference panel of frequently observed MHC-I and MHC-II alleles. Predicted peptides were ranked based on $\mathrm{IC}_{50}$ and IFN- $\gamma$ Inducer Scores, respectively, and top performing epitopes were synthesized and subjected to in vitro screening by IFN- $\gamma$ enzyme-linked immunosorbent spot assay (ELISpot). Independent trials were conducted using PBMCs of healthy volunteers. Final chosen peptides were fused with flexible GS linkers in silico to design a novel polypeptide vaccine.

Results: Seven immunodominant peptides screened from 44 predicted peptides were included in the vaccine design, selected to elicit specific immune responses across MHC class I and class II, and across HPV types. Evaluation of the vaccine's properties suggest that the vaccine is stable, non-allergenic, and provides near complete global population coverage ( $>99 \%)$. Solubility prediction and rare codon analysis indicate that the DNA sequence encoding the vaccine is suitable for high level expression in Escherichia coli.

50 Conclusions: In sum, this study demonstrates the potential and lays the framework for the 51 development of a peptide-based vaccine against warts. 


\section{Introduction}

Skin warts (verrucae) are benign lesions in the cutaneous epithelium. They are caused by the human papillomavirus (HPV), a small, non-enveloped, DNA virus that is easily transmitted through skin-to-skin contact [1]. Peak incidence of wart infection occurs in schoolchildren aged $12-16$, where prevalence can be as high as $20 \%$ [2]. While two-thirds of warts regress spontaneously within a few years, they may cause significant morbidity in affected individuals. Occasional reports have even shown warts becoming malignant and progressing into verrucous carcinoma [3].

While there are more than 200 HPV genotypes, only a handful are responsible for cutaneous warts. Common warts are most frequently associated with HPV types 1, 2, and 7, plantar warts with types 1 and 2, and flat warts with types 3 and 10. Other higher-risk HPV types are oncogenic and are a principal cause of cervical cancer [4, 5]. Differentiation of HPV types is based on the nucleotide sequence of the L1 major protein [6]. The L1 protein, along with the L2 minor component, forms pentameric capsomer subunits that make up the capsid shell of the virus. L1 has been shown to spontaneously self-assemble into virus-like particles (VLPs) that can act as highly potent immunogens, presenting an exterior surface nearly indistinguishable from the native virion [7]. As such, the L1 protein has been widely used in existing VLP-based HPV vaccines and is the target for novel subunit vaccines.

Thus far, vaccine development efforts for HPV have been centered on higher-risk HPV types. There are currently 3 licensed VLP-based HPV vaccines: Cervarix ${ }^{\circledR}$ bivalent vaccine, and Gardasil ${ }^{\circledR}$ Quadrivalent and 9-valent vaccine [8]. These vaccines all possess a prophylactic effect against HPV types 16 and 18, which together account for approximately $70 \%$ of cervical cancer cases [9]. Because VLPs lack the viral genome but have a morphologically similar outer structure to the virus, they are both noninfectious and highly immunogenic. Recent studies have also sought to develop effective therapeutic HPV vaccines, as well as vaccines that improve accessibility to developing countries [10-13]. In comparison, little attention has been afforded to vaccine development for low-risk HPV types. To date, no effective antiviral drugs are available for wart treatment.

Instead, existing treatment modalities are largely aimed at destruction of wart tissue. Cryotherapy by liquid nitrogen is the most common approach and involves inducing cold injury to warts [14]. Freezing treatment is repeated every 2-3 weeks until warts have cleared. Salicylic acid application is also commonly used to promote exfoliation of epidermal cells and is postulated to have an added mechanism of stimulating host immunity [15]. Studies have proven salicylic acid to be somewhat more effective than placebo [16]. Nonetheless, conventional treatments can be painful and inefficient, with low clearance rates and high recurrence rates [17]. Moreover, treatment has the potential to cause moderate to severe scarring, leading to severe deficits in cosmesis [3]. These risks have led many physicians to opt for a wait-and-see approach, leaving patients with cosmetic insecurity and physical discomfort. Others have turned to 
alternative techniques such as laser therapy and oral isotretinoin, but issues over recurrent infection remain [17-19]. Evidently, there is a need for a prophylactic, nondestructive wart treatment. We observe that the creation of a treatment with these properties can be achieved by adapting the vaccine design approaches currently employed for higher-risk HPV types.

This study aims to identify immunodominant T-lymphocyte epitopes from the L1 protein of HPV 1, 2 and 3, as the first step for bioengineering a peptide-based vaccine for warts. In recent years, peptides have become increasingly attractive vaccine candidates with the potential to overcome the disadvantages of traditional vaccines, including those based on VLPs.

98 Compared to vaccines using eukaryotic expression systems, peptide-based vaccines are easily produced in Escherichia coli, and their greater thermal stability eliminates the need for cold chain [20]. The lack of redundant elements also minimizes unwanted allergic reactions and allows the vaccine to be tailored for more focused immune responses [21, 22]. Beyond these advantages, the inclusion of multiple epitopes allows for a single peptide vaccine to target several strains, which is crucial to maximizing coverage across wart-causing HPV types with low sequence similarity [23].

Epitope selection is the first and most crucial stage in designing peptide-based vaccines. Effective epitopes must be able to induce high levels of humoral and cellular immune responses. In this study, we employ a peptide screening method relying on immunoinformatic tools that predict MHC-I and MHC-II binding affinity, followed by in-vitro testing by enzyme-linked immunosorbent spot (ELISpot) assay [24]. This two-step process reduces the number of experiments needed, thereby facilitating efficient candidate epitope identification. Following insilico screening, ELISpot assay was performed to evaluate the response of human $\mathrm{CD}^{+}$and

$112 \mathrm{CD}^{+} \mathrm{T}$-lymphocytes to the peptide stimuli. The assay provides quantitative measurements of the 113 secretion of IFN- $\gamma$, an abundant cytokine produced by Th1 cell with a critical role in activation 114 of innate and adaptive immunity. IFN- $\gamma$ ELISpot assays are used extensively for screening of 115 immune responses in vaccine development and have been shown to be both sensitive and 116 reproducible $[25,26]$. 


\section{Materials and Methods}

\subsection{Sequence Retrieval}

The amino acid sequences of the L1 protein for HPV 1a, 2, and 3 were retrieved from the

121 National Center for Biotechnology Information database (http://www.ncbi.nlm.nih.gov/) with

122 accession numbers of CAA01195.1, ABO14919.1, and P36731.1, respectively.

125 due to low sequence similarity among the HPV types. The prediction process is summarized by the flowchart depicted in Figure 1.

\section{MHC-I \& MHC-II binding prediction \\ Input proteins}

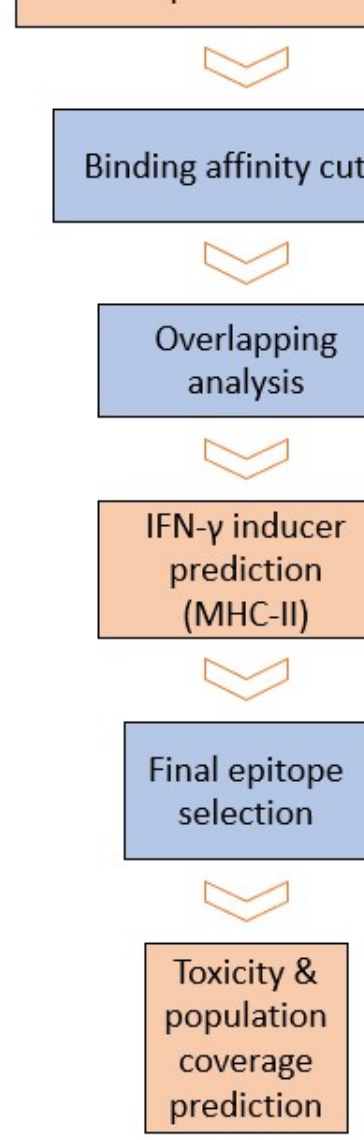

129 Fig 1. Flowchart for bioinformatics analysis in the study. The process was repeated to predict epitopes for HPV 1, 2, and 3. 


\subsubsection{MHC Class I Epitope Prediction}

MHC-I binding epitope prediction was performed using three methods from the IEDB (www.iedb.org) database (Table 1). Predictions of 8-11 mer epitope binding sites were made against a reference panel of 27 frequently observed MHC-I HLA alleles, with a reported population coverage greater than 97\% [27]. Predicted epitopes were ranked according to their half maximal inhibitory concentration ( $\mathrm{IC}_{50}$ ) scores, and peptides below the MHC-I allele specific affinity cutoff thresholds were excluded from further analysis [28, 29]. From this filtered list, overlapping sequences from the three prediction methods were selected.

\subsubsection{MHC Class II Epitope Prediction}

Two servers, IEDB and NetMHCIIpan 3.2, were used for prediction of 15 mer MHC-II binding epitopes (Table 1). A similar reference panel of 27 MHC-II HLA alleles with a population coverage greater than $99 \%$ was used for analysis [30]. Predicted peptides with an adjusted percentile rank greater than 10 were filtered out, and subsequent overlapping analysis of the results of the two prediction methods was performed.

Table 1. Methods used for T-cell epitope binding prediction.

\begin{tabular}{|c|c|c|c|c|}
\hline & Server & Link & Prediction Method & References \\
\hline \multirow{3}{*}{$\begin{array}{l}\text { MHC-I } \\
\text { Binding }\end{array}$} & \multirow[t]{3}{*}{ IEDB } & \multirow[t]{3}{*}{$\underline{\text { http://tools.iedb.org/mhci/ }}$} & NetMHC (ANN) $4.0-$ ANNs & {$[31-36]$} \\
\hline & & & NetMHCI Pan BA $4.0-$ ANNS & [37-41] \\
\hline & & & $\begin{array}{l}\text { PickPocket - binding specificities based on } \\
\text { receptor pocket similarities }\end{array}$ & [42] \\
\hline \multirow[t]{2}{*}{$\begin{array}{l}\text { MHC-II } \\
\text { Binding }\end{array}$} & IEDB & http://tools.iedb.org/mhcii/ & $\begin{array}{l}\text { IEDB Recommended } 2.22 \text { - consensus } \\
\text { approach combining ANN, SMM, } \\
\text { combinatorial library, and pocket profiles }\end{array}$ & [43-49] \\
\hline & $\begin{array}{l}\text { NetMHCIIpa } \\
\mathrm{n}\end{array}$ & $\begin{array}{l}\text { http://www.cbs.dtu.dk/servic } \\
\underline{\text { es/NetMHCIIpan-3.2/ }}\end{array}$ & NetMHCIIpan 3.2 - ANNs & [43] \\
\hline
\end{tabular}

\subsubsection{Evaluation of Selected Epitopes}

Overlapping MHC-I and MHC-II epitopes were reranked based on mean $\mathrm{IC}_{50}$ and IFN- $\gamma$ Inducer Score, respectively. The potential of MHC-II epitopes to induce IFN- $\gamma$ production was evaluated with the IFNepitope server (https://crdd.osdd.net/raghava/ifnepitope), using a Support Vector Machines (SVMs) based method [50]. This study recognizes the importance of $\mathrm{CD}^{+}$cell activation in vaccine formulation. Accordingly, $15 \mathrm{MHC}-\mathrm{II}$ binding peptides with the highest IFN- $\gamma$ Inducer Score, and 5 MHC-I binding peptides with the lowest mean $\mathrm{IC}_{50}$ were chosen for both HPV type 1 and 2 . Only 5 peptides with the highest binding affinities were selected from HPV type 3, in accordance with the strain's relatively lower prevalence of infection. Lastly, the toxicity (http://crdd.osdd.net/raghava/toxinpred/multi submit.php) of the chosen peptides were assessed, and peptides with a positive toxicity prediction were excluded [51]. In total, 45 peptides were elected for in vitro testing. 


\subsection{Peptide Synthesis}

Peptide sequences were synthesized by Kelowna International Scientific (Taiwan) with more than $90 \%$ purity. Peptides were received in lyophilized form, and stock solutions were created by dissolving $40 \mathrm{mg} / \mathrm{mL}$ in DMSO. Peptides were stored at $-20{ }^{\circ} \mathrm{C}$ until use, where they were diluted to $1 \mathrm{mg} / \mathrm{mL}$ in PBS.

\subsection{In vitro Immunodominant Peptides Screening}

To screen the candidate peptides, IFN- $\gamma$ ELISpot assay (Mabtech) was performed using PBMCs of healthy human volunteers, in 4 independent trials. Isolated T-cells were seeded $2 \mathrm{x}$ $10^{5}$ in $200 \mu \mathrm{L}$ medium (RPMI, 5\% AB serum, 1\% PSA) with IL-2 and IL-7 cytokines in round bottom 96 well plates. $4 \mu \mathrm{L}$ of peptide solution or PBS control was added to the wells, so that each peptide was present in 2 wells. Dendritic cells (DC) in $15 \mathrm{~mL}$ medium were seeded in a T75 cell culture flask. Cultured cells were incubated at $37^{\circ} \mathrm{C}$. Cell medium was changed and peptides were added every 2 days. After 7 days, following DC maturation, T-cells and DCs were cocultured in a 10:1 ratio. Cells were cultured for another 6 days.

ELISpot PVDF plates (MSIPS4510, Millipore) were activated with $30 \mu \mathrm{L} \mathrm{35 \%} \mathrm{EtOH} \mathrm{for}$ 1 minute. Following washing, $100 \mu \mathrm{L}$ of $15 \mu \mathrm{g} / \mathrm{mL} 1-\mathrm{D} 1 \mathrm{~K}$ coating antibody was added to each well and allowed to react overnight. After blocking with $200 \mu \mathrm{L}$ medium with $10 \%$ AB serum for $30 \mathrm{~min}$, cells were seeded $1 \times 10^{5}$ per well and stimulated with $8 \mu \mathrm{L}$ peptide, $2 \mu \mathrm{L} \mathrm{OKT-3}$, or $8 \mu \mathrm{L}$ medium. Spots were detected using a biotinylated antibody (7-B6-1-biotin) against streptavidin conjugated ALP and visualized by the addition of a colorimetric substate (BCIP/NBT).

Plates were scanned and the number of spot forming cells (SFCs) in each well were counted by an ImmunoSpot reader (CTL Cellular Technology). The stimulation index of each peptide was calculated as the ratio of SFCs in peptide-stimulated cells to that of non-stimulated cells. Peptides with an SI value greater than 2 were regarded as positive and immunodominant.

\subsection{Design of Recombinant Polypeptide Vaccine and In Silico Cloning}

Of the peptides that passed ELISpot screening, 7 were included in the design of a polypeptide HPV 1, 2, and 3 vaccine. Peptides were fused with a flexible glycine-serine linker (GS), as studies have reported they provide the best structure and stability for fusion proteins [52]. The physiochemical properties, including stability, molecular weight, and isoelectric point, of the resulting polypeptide vaccine were evaluated using ProtParam server (https://web.expasy.org/protparam/), and the vaccine's global population coverage was analyzed using the IEDB tool (http://tools.iedb.org/population/) [51, 53]. Protein solubility upon expression in E. Coli was predicted using the ccSOL omics server (http://s.tartaglialab.com/page/ccsol_group), with a reported 74\% accuracy [54]. Finally, AllergenFP (http://ddg-pharmfac.net/AllergenFP/) was used to ensure the vaccine had no allergenic properties [55]. 
197 To prepare for expression in E. coli, the polypeptide sequence was reverse translated. A 198 hexahistidine tag (6x-His) was attached upstream of the vaccine sequence for ease of purification, 199 and the entire sequence was codon optimized through the EMBOSS backtranseq tool

200 (https://www.ebi.ac.uk/Tools/st/emboss_backtranseq/) [56]. To verify the potential for high level 201 protein expression, the optimized DNA sequence was evaluated for codon adaptation index

202 (CAI), GC content, and codon frequency and distribution (CFD) using the GenScript Rare

203 Codon Analysis Tool (https://www.genscript.com/tools/rare-codon-analysis) [13]. Finally,

204 restriction sites of EcoRI and XbaI were inserted at the start of the sequence, while SpeI and PstI 205 were inserted at the end. 


\section{3. Results}

\subsection{T-cell Epitope Prediction}

209 In the first phase of this study, a variety of bioinformatics tools were employed to predict 210 and filter T-cell epitopes from the L1 protein of wart-causing HPV strains (Table 2). During this 211 prediction phase, an emphasis was placed on binding affinity, as it is both an important factor for

212 vaccine construction and can be reliably predicted by existing tools. MHC-I and MHC-II binding 213 predictions were consolidated from multiple methods to ensure accurate predictions. Of the 45

214 highest scoring candidate peptides, 44 were successfully synthesized and subjected to in vitro 215 screening. 
217 Table 2. Predicted epitopes from HPV 1, 2, and 3 L1 major capsid proteins.

\begin{tabular}{|c|c|c|c|c|c|c|}
\hline $\begin{array}{l}\text { Protein } \\
\text { Source }\end{array}$ & Peptide ID & Epitope & Location & $\begin{array}{l}\text { Mean } \\
\text { IC }_{50}\end{array}$ & $\begin{array}{c}\text { IFN- } \gamma \\
\text { Inducer } \\
\text { Score }\end{array}$ & $\begin{array}{c}\text { Toxin } \\
\text { Prediction }\end{array}$ \\
\hline \multirow{20}{*}{ HPV1a-L1 } & pHPV1a-1 & QLFNRSYWL & $312-320$ & 19.83 & - & Non-toxin \\
\hline & pHPV1a-2 & FQMAVWLPA & $5-13$ & 19.97 & - & Non-toxin \\
\hline & pHPV1a-3 & ILEDWQLSV & $403-412$ & 21.61 & - & Non-toxin \\
\hline & pHPV1a-4 & RLVWGLRGI & $101-111$ & 23.26 & - & Non-toxin \\
\hline & pHPV1a-5 & KLTPENLAYI & $388-397$ & 28.49 & - & Non-toxin \\
\hline & pHPV1a-6 & PNAFRVFRVRFADPN & $71-85$ & 44.17 & 1.43 & Non-toxin \\
\hline & pHPV1a-7 & NAFRVFRVRFADPNR & $72-86$ & 43.99 & 1.19 & Non-toxin \\
\hline & pHPV1a-8 & EDQYRFLGSSLAAKC & $421-435$ & 5.77 & 1.17 & Non-toxin \\
\hline & pHPV1a-9 & KYPDYIRMNHEAYGN & $234-248$ & 155.58 & 1.14 & Non-toxin \\
\hline & pHPV1a-10 & YPDYIRMNHEAYGNS & $235-249$ & 155.58 & 1.14 & Non-toxin \\
\hline & pHPV1a-11 & TNYVGTPSGSMVSSD & $296-310$ & 66.93 & 1.11 & Non-toxin \\
\hline & pHPV1a-12 & FRVFRVRFADPNRFA & $74-88$ & 30.90 & 1.11 & Non-toxin \\
\hline & pHPV1a-13 & YRFLGSSLAAKCPEQ & $424-438$ & 11.71 & 1.10 & Non-toxin \\
\hline & pHPV1a-14 & IPKVSPNAFRVFRVR & $66-80$ & 140.33 & 1.05 & Non-toxin \\
\hline & pHPV1a-15 & CKYPDYIRMNHEAYG & $233-247$ & 192.18 & 1.02 & Non-toxin \\
\hline & pHPV1a-16 & VFRVRFADPNRFAFG & $76-90$ & 33.30 & 1.02 & Non-toxin \\
\hline & pHPV1a-17 & VSPNAFRVFRVRFAD & $69-83$ & 42.88 & 0.94 & Non-toxin \\
\hline & pHPV1a-18 & RFLGSSLAAKCPEQA & $425-439$ & 83.09 & 0.93 & Non-toxin \\
\hline & pHPV1a-19 & KVSPNAFRVFRVRFA & $68-82$ & 30.47 & 0.93 & Non-toxin \\
\hline & pHPV1a-20 & AFRVFRVRFADPNRF & $73-87$ & 28.62 & 0.90 & Non-toxin \\
\hline \multirow[t]{20}{*}{ HPV2-L1 } & pHPV2-1 & LLEDWNFGV & $408-416$ & 8.61 & - & Non-toxin \\
\hline & pHPV2-2 & QLLEDWNFGV & $407-416$ & 9.98 & - & Non-toxin \\
\hline & pHPV2-3 & RLLWACVGV & $112-120$ & 10.69 & - & Non-toxin \\
\hline & pHPV2-4 & FLLQRGAMPTV & $478-488$ & 10.70 & - & Non-toxin \\
\hline & pHPV2-5* & LLWACVGVEV & $113-122$ & 14.85 & - & Non-toxin \\
\hline & pHPV2-6 & KVISTDVYVTRTNVY & $36-50$ & 131.60 & 1.06 & Non-toxin \\
\hline & pHPV2-7 & VSTISLQMALWRPNE & $10-24$ & 158.97 & 0.73 & Non-toxin \\
\hline & pHPV2-8 & SKVISTDVYVTRTNV & $35-49$ & 109.27 & 0.71 & Non-toxin \\
\hline & pHPV2-9 & STDVYVTRTNVYYHG & $39-53$ & 97.08 & 0.70 & Non-toxin \\
\hline & pHPV2-10 & LQRGAMPTVSRKRAA & $480-494$ & 121.62 & 0.65 & Non-toxin \\
\hline & pHPV2-11 & TNVYYHGGSSRLLTV & $47-61$ & 23.23 & 0.63 & Non-toxin \\
\hline & pHPV2-12 & TPVSKVISTDVYVTR & $32-46$ & 158.19 & 0.62 & Non-toxin \\
\hline & pHPV2-13 & STISLQMALWRPNES & $11-25$ & 75.85 & 0.61 & Non-toxin \\
\hline & pHPV2-14 & NVSTISLQMALWRPN & $9-23$ & 52.94 & 0.61 & Non-toxin \\
\hline & pHPV2-15 & LLQRGAMPTVSRKRA & $479-493$ & 73.58 & 0.60 & Non-toxin \\
\hline & pHPV2-16 & PVSKVISTDVYVTRT & $33-47$ & 113.65 & 0.59 & Non-toxin \\
\hline & pHPV2-17 & TQRLLWACVGVEVGR & $110-124$ & 816.94 & 0.54 & Non-toxin \\
\hline & pHPV2-18 & VSRKRAAVSGTTPPT & $488-502$ & 67.34 & 0.54 & Non-toxin \\
\hline & pHPV2-19 & RTNVYYHGGSSRLLT & $46-60$ & 19.18 & 0.53 & Non-toxin \\
\hline & pHPV2-20 & YYHGGSSRLLTVGHP & $50-64$ & 75.37 & 0.49 & Non-toxin \\
\hline \multirow[t]{5}{*}{ HPV3-L1 } & pHPV3-1 & EEYDLQFIFQLCK & $397-409$ & - & - & Non-toxin \\
\hline & pHPV3-2 & LPDPNKFGLPDA & $105-116$ & - & - & Non-toxin \\
\hline & pHPV3-3 & GVEVGRGLPLGVGL & $132-145$ & - & - & Non-toxin \\
\hline & pHPV3-4 & QFPLGRKFLMQ & $490-500$ & - & - & Non-toxin \\
\hline & pHPV3-5 & RLLTVGHP & $68-76$ & - & - & Non-toxin \\
\hline
\end{tabular}




\subsection{In vitro Immunodominant Peptides Screening}

b)

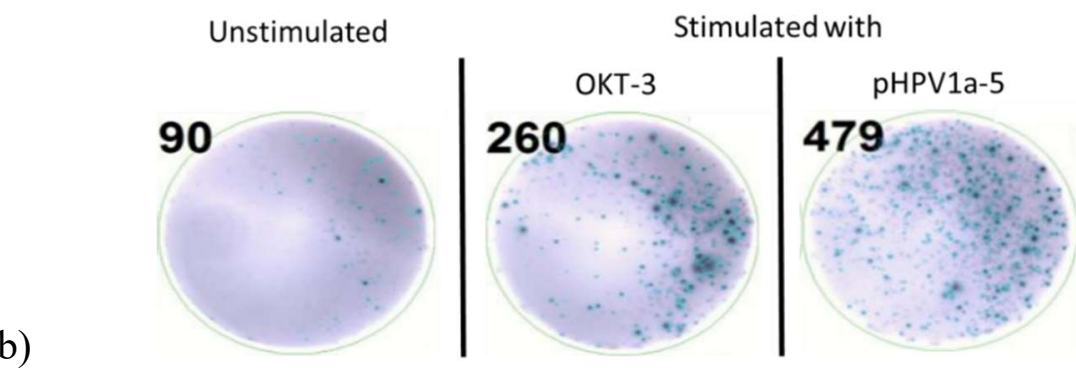
HPV 2, and 1 for HPV 3.

a)

\section{Peptides}

Elected peptides were subjected to screening by IFN- $\gamma$ ELISpot assay, where cells were stimulated with PBS, peptides, or OKT-3 positive control. Results from independent trials of 4 human volunteers were averaged. Of the 44 peptides screened, 11 produced an SI value greater than 2 and were therefore considered immunodominant (Fig. 2). The distribution of targets for the immunodominant peptides were 1 for MHC-I HPV 1a, 7 for MHC-II HPV 1a, 2 for MHC-II

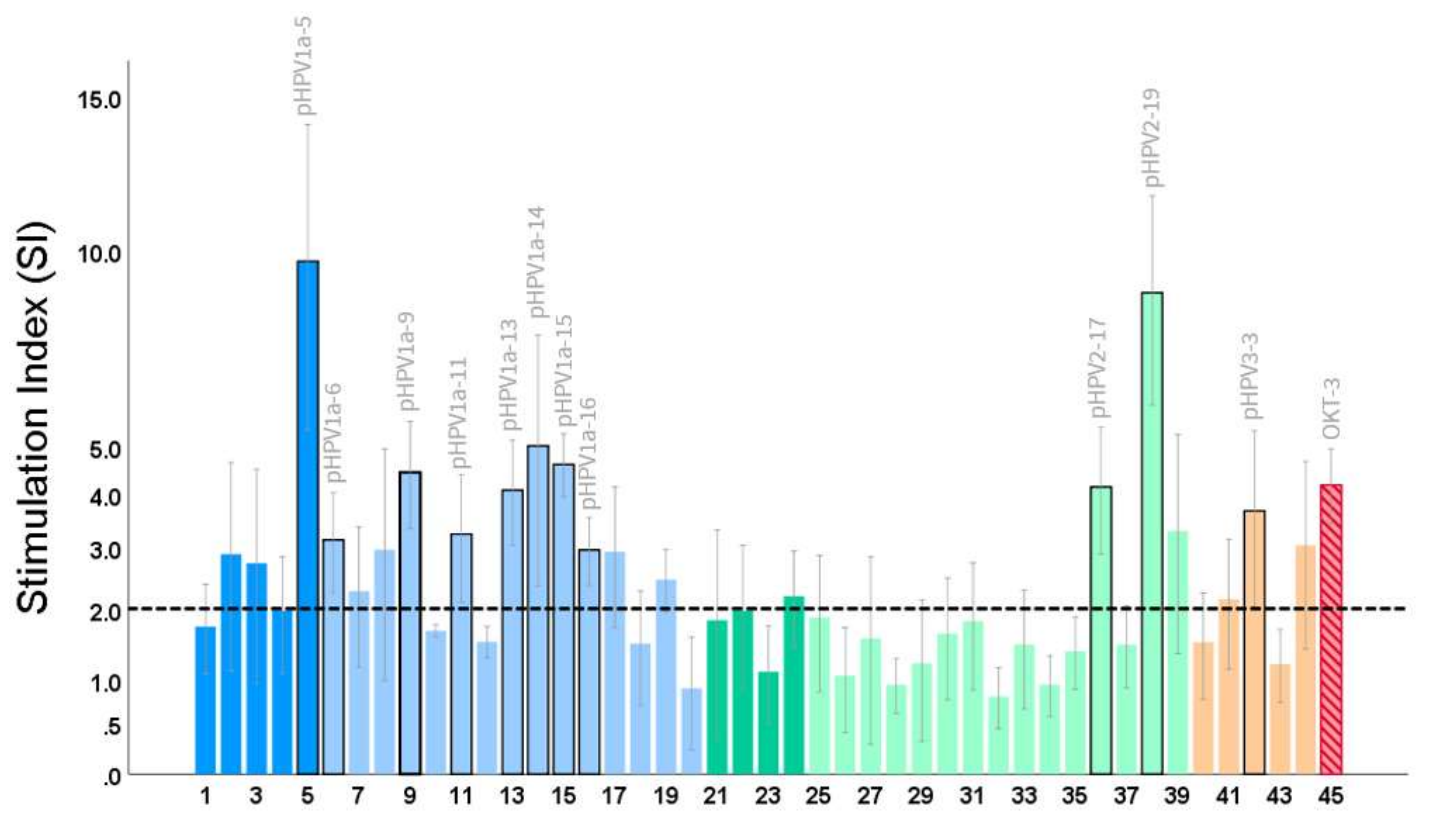

Fig. 2. Immunodominant peptides screening via ELISpot assay. a) PBMCs obtained from healthy human volunteers were stimulated with peptides selected from the L1 protein of HPV 1a (blue), HPV 2 (green), and HPV 3 (orange). The stimulation index was calculated as the ratio of SFCs in peptide stimulated cells and PBS mediums-stimulated cells. The threshold for immunodominance was set at SI $>2$, as labeled in the figure. b) A representative image of wells analyzed by a CTL ImmunoSpot reader is shown, illustrating the number of spots observed in a non-stimulated well, an OKT-3 stimulated well, and a well stimulated by an immunodominant peptide, pHPV1a-5. 


\subsection{Sequence Selection for Vaccine Design}

In preparation for vaccine design, further screening of the $7 \mathrm{MHC}-\mathrm{II}$ epitopes for HPV 1a was performed to reduce redundancy. Using the IEDB population coverage prediction tool (http://tools.iedb.org/population/), it was shown that peptides pHPV1a-6 and pHPV1a-15 had global population coverages of $96.90 \%$ and $77.19 \%$, respectively, significantly higher than the other 5 peptide sequences. Thus, they were chosen for the vaccine. Next, the lack of immunodominant MHC-I epitopes targeting HPV 2 was resolved by the inclusion of pHPV2-4 in the vaccine. While the mean SI of pHPV2-4 fell slightly below the threshold, the peptide yielded positive results in 2 of the 4 subjects. Ultimately, 7 peptides were included in the final vaccine design (Table 3).

Table 3. Peptides selected for vaccine design.

\begin{tabular}{|c|c|c|c|c|c|c|c|}
\hline \multirow{2}{*}{$\begin{array}{l}\text { Peptide } \\
\text { ID }\end{array}$} & \multirow[t]{2}{*}{ Epitope } & \multirow[t]{2}{*}{ Location } & \multicolumn{4}{|c|}{ Stimulation Index } & \multirow{2}{*}{$\begin{array}{c}\text { Predicted MHC } \\
\text { Alleles }\end{array}$} \\
\hline & & & Subject 1 & Subject 2 & Subject 3 & Subject 4 & \\
\hline pHPV1a-5 & KLTPENLAYI & $388-397$ & 21.3 & 11.3 & 2.3 & 4.0 & $\begin{array}{l}\text { HLA-A } \text { A }^{*} 2: 03, \mathrm{HLA}-\mathrm{A}^{*} 02: 01, \\
\text { HLA-A } 02: 06, \mathrm{HLA}-\mathrm{A}^{*} 68: 02\end{array}$ \\
\hline pHPV1a-6 & PNAFRVFRVRFADPN & $71-85$ & 2.5 & 3.3 & 1.3 & 5.5 & 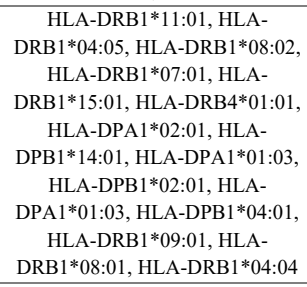 \\
\hline pHPV1a-13 & YRFLGSSLAAKCPEQ & $424-438$ & 7.0 & 3.7 & 2.0 & 3.7 & $\begin{array}{c}\text { HLA-DQA1*05:01, HLA- } \\
\text { DQB1*03:01, HLA-DPA }{ }^{*} 02: 01, \\
\text { HLA-DPB1*14:01, HLA- } \\
\text { DRB1*04:01, HLA-DRB }{ }^{*} 01: 01, \\
\text { HLA-DRB1*01:01 }\end{array}$ \\
\hline pHPV2-17 & TQRLLWACVGVEVGR & $110-124$ & 4.8 & 1.6 & 2.9 & 7.4 & $\begin{array}{l}\text { HLA-DQA1*04:01, HLA- } \\
\text { DQB1*04:02 }\end{array}$ \\
\hline pHPV2-19 & RTNVYYHGGSSRLLT & $46-60$ & 7.3 & 16.2 & 9.3 & 2.4 & 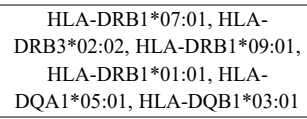 \\
\hline pHPV3-3 & GVEVGRGLPLGVGL & $132-145$ & 1.3 & 0.56 & 7.4 & 5.5 & $\begin{array}{l}\text { HLA-DRB1*09:01, HLA- } \\
\text { DQA1*05:01, HLA-B*A* } \\
\text { HLA-A } * 6: 02: 02\end{array}$ \\
\hline pHPV2-4* & FLLQRGAMPTV & $478-488$ & 3.5 & 1.5 & 0.43 & 3.4 & $\begin{array}{l}\text { HLA-A }{ }^{*} 02: 01, \text { HLA-A**02:06, } \\
\text { HLA-A } \text { A }^{*} 02: 03\end{array}$ \\
\hline
\end{tabular}

Note. - *While the mean SI of pHPV-4 was not positive $(>2)$, it was included in the vaccine design for coverage of MHC-I binding of HPV 2. Moreover, pHPV-4 did produce SI $>2$ in 2 of the 4 subjects.

\subsection{Design of Recombinant Polypeptide Vaccine and In Silico Cloning}

Figure 3 illustrates the construct of the candidate polypeptide vaccine. Inclusion of multiple peptides allows for simultaneous coverage of HPV types 1, 2, and 3. Results from server analysis of the vaccine's physiochemical properties show that it has a molecular weight of $12.04 \mathrm{kDa}$, a theoretical $\mathrm{pI}$ of 10.27 , and an instability index of 39.70 , indicating that the vaccine is stable (Table 4). Furthermore, the vaccine is also predicted to have high global population 
258 coverage $(99.63 \%)$, and decent solubility propensity (54\%). The vaccine is classified as a non259 allergen.

Following reverse translation, the codon optimized gene for the vaccine was evaluated 261 for codon adaptation index, GC content, and codon frequency distribution with $E$. Coli selected 262 as the expression host (Fig. 4). Results from these parameters indicate the sequence is suitable 263 for cloning and expression in E. coli.

264

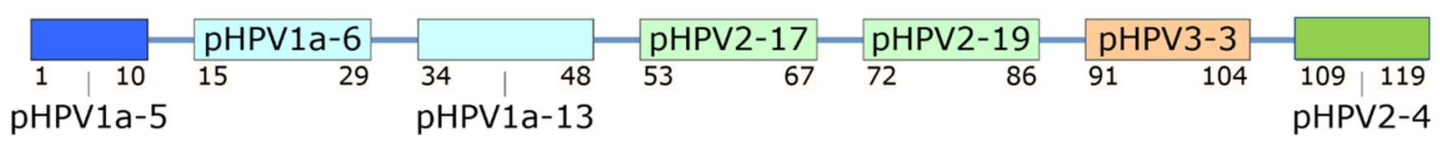

265

266

267

268

269

Table 4. Physiochemical properties, population coverage, and allergenicity of recombinant vaccine.

\section{Vaccine Properties}

Molecular weight (Da)

No. of amino acids

Instability index

Gravy

Aliphatic index

Theoretical pI

Solubility propensity

Global population coverage

Allergenicity
12044.63

119

39.70 (Stable)

$-0.062$

76.13

10.27

$54 \%$

99.63\%

Probable Non-Allergen
KLTPENLAYIGGGSPNAFRVFRVRF

ADPNGGGSYRFLGSSLAAKCPEQG

GGSTQRLLWACVGVEVGRGGGSR

TNVYYHGGSSRLLTGGGSGVEVGR

GLPLGVGLGGGSFLLQRGAMPTV 
a)

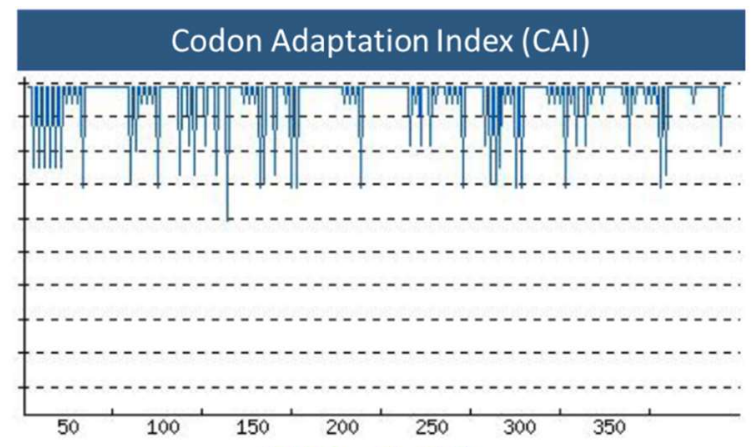

CAl: 0.90

\section{GC Content Adjustment}

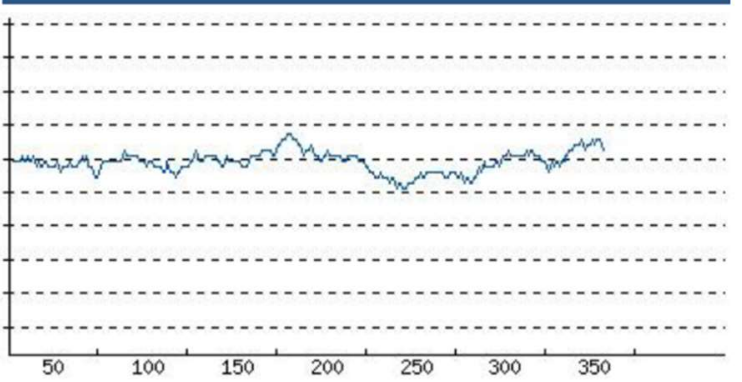

b) Average GC Content: $58.08 \%$

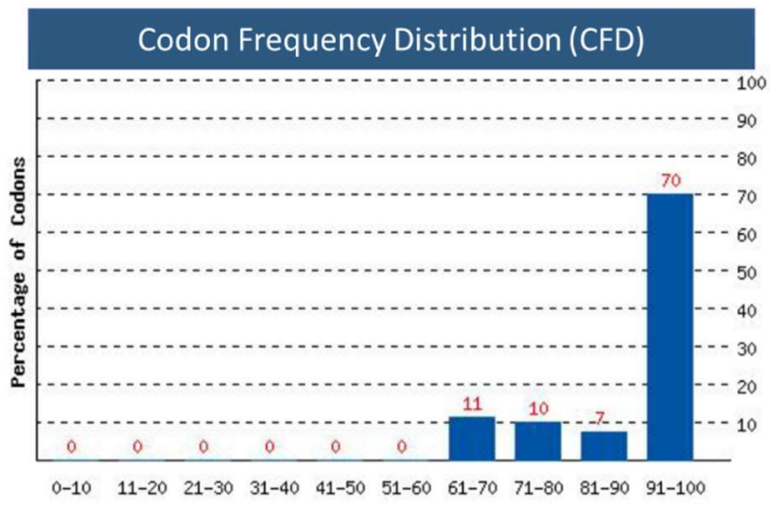

272 Fig. 4. Evaluation of parameters for high-level protein expression in E. coli. a) The sequence has

273 a CAI value of 0.90 . CAI is the distribution of codon usage frequency along the length of the

274 coding region, and a value $>0.8$ is considered good for expression. b) The average GC content of

275 the sequence is $58.08 \%$, and there are no peaks outside of the ideal range of $30-70 \%$. c) The

276 usage of codons with frequency scores lower than 30 are likely to adversely affect expression

277 efficiency. The percentage of low frequency codons in this sequence is $0 \%$. 


\section{Discussion}

Existing wart treatments have low clearance rates and high recurrence rates, and as such, are increasingly seen as non-viable options for recalcitrant infections [17]. Licensed VLP-based HPV vaccines on the market target high-risk oncogenic HPV types, and while studies have reported limited positive responses against anogenital and cutaneous warts, their efficacy is hindered by a lack of specific coverage for verrucae-causing types, namely, HPV types 1, 2, and $3[57,58]$.

In this study, we aim to repurpose the peptide-based vaccine design methods currently employed for high-risk HPV types to develop a novel, cutaneous wart vaccine. The value of such an approach is derived from its therapeutic and prophylactic effects, its non-destructive nature, as well as its ease of production and transport compared to traditional vaccines [20]. Furthermore, by eliminating the need for a strict treatment regimen, the approach holds the potential to relieve patients and physicians from the frustrations caused by problematic warts [59].

The proposed recombinant polypeptide vaccine is the product of a peptide screening process combining both immunoinformatics and in vitro assays. In recent years, the fields of genomics and bioinformatics have evolved to enable computational screening of epitopes considering a diversity of HLAs [60]. The reliability of such methods can be increased by analyzing the results from multiple prediction tools. This study uses immunoinformatic tools to narrow the epitope pool to a manageable number for in vitro tests, to facilitate an efficient and accurate experimental design.

Using this process, the final vaccine design consists of 7 T-lymphocyte epitopes, including both CTL and T-helper cell epitopes, selected for their immunodominance via human IFN- $\gamma$ ELISpot assays. The resultant vaccine is multi-target, with epitopes designed to elicit specific immune responses to HPV types 1, 2, and 3. Predictions using bioinformatics tools suggest that the vaccine is stable, non-allergenic, and provides near total global population coverage (>99\%). Because L1 major capsid protein plays a crucial role in HPV infection and its immunogenicity is well documented, it was selected as the source protein in this study. However, the L2 minor capsid protein, inducing low-titer yet cross-neutralizing antibodies, has also been proposed as an alternative to develop broader-spectrum HPV vaccines, and may be a point worth investigating in the future [61].

To ensure high levels of recombinant expression in prokaryotic systems, the GenScript tool was used to confirm the full-length polypeptide vaccine's suitability for expression in E. coli host [13]. The vaccine's decent solubility propensity score also indicates less accumulation of proteins in the inclusion bodies. With the addition of restriction enzymes and a N-terminal histag, the DNA sequence encoding the vaccine was prepared for further studies, which consist of transforming the recombinant plasmid into $E$. coli and performing subsequent tests for immunogenicity [62]. 
316 By identifying immunodominant epitopes, this study provides the basis for the

317 development of a peptide-based wart vaccine. However, important questions about enhancement

318 and delivery must be settled sooner or later in the vaccine engineering process. It is known that

319 subunit peptide vaccines can have limited immunogenicity. As such, the incorporation of

320 immunostimulatory adjuvants has often been utilized to enhance vaccine responses [21].

321 Choosing an ideal adjuvant can help improve innate and acquired immunity, so careful selections

322 should be made based on the host, pathogen, vaccine antigen, and the route of immunization [63].

323 The arrangement of epitopes and adjuvants should also be subjected to in silico optimization [64].

324 Appropriate consideration of these factors are needed, should the findings in this study be built

325 upon in the future. 


\section{Conflict of Interest}

328 The authors declare that the research was conducted in the absence of any commercial or financial 329 relationships that could be construed as a potential conflict of interest.

332 The datasets generated for this study are available on request to the corresponding author. Author Contributions WHT and WHC conceptualized the study. WHT performed the experiments. WHT, CWW, and 336 WHC analyzed the data. WHC and CWW supervised the study. WHT wrote the first draft of the 337 manuscript. All authors contributed to the article and approved the submitted version. 


\section{References}

1. Human Papillomaviruses, in IARC Monographs on the Evaluation of Carcinogenic Risks to Humans. 2007, International Agency for Research on Cancer: Lyon, France.

2. Kilkenny, M. and R. Marks, The descriptive epidemiology of warts in the community. Australas J Dermatol, 1996. 37(2): p. 80-6.

3. $\quad$ Nigam, A.M.A.A.P.K., Wart. 2020, StatPearls Treasure Island, Florida

4. $\quad$ Okunade, K.S., Human papillomavirus and cervical cancer. J Obstet Gynaecol, 2020. 40(5): p. 602-608.

5. Steele, K., et al., A study of HPV 1, 2 and 4 antibody prevalence in patients presenting for treatment with cutaneous warts to general practitioners in N. Ireland. Epidemiol Infect, 1988. 101(3): p. 537-46.

6. Bzhalava, D., C. Eklund, and J. Dillner, International standardization and classification of human papillomavirus types. Virology, 2015. 476: p. 341-344.

7. Buck, C.B., P.M. Day, and B.L. Trus, The papillomavirus major capsid protein L1. Virology, 2013. 445(1-2): p. 169-74.

8. Pouyanfard, S. and M. Müller, Human papillomavirus first and second generation vaccinescurrent status and future directions. Biol Chem, 2017. 398(8): p. 871-889.

9. Braaten, K.P. and M.R. Laufer, Human Papillomavirus (HPV), HPV-Related Disease, and the HPV Vaccine. Rev Obstet Gynecol, 2008. 1(1): p. 2-10.

10. Namvar, A., et al., Development of HPV $(16,18,31,45)$ E5 and E7 peptides-based vaccines predicted by immunoinformatics tools. Biotechnol Lett, 2020. 42(3): p. 403-418.

11. Panahi, H.A., et al., A comprehensive in silico analysis for identification of therapeutic epitopes in HPV16, 18, 31 and 45 oncoproteins. PLoS One, 2018. 13(10): p. e0205933.

12. Vici, P., et al., Targeting immune response with therapeutic vaccines in premalignant lesions and cervical cancer: hope or reality from clinical studies. Expert Rev Vaccines, 2016. 15(10): p. 1327-36.

13. Yazdani, Z., et al., Designing a potent L1 protein-based HPV peptide vaccine: A bioinformatics approach. Comput Biol Chem, 2020. 85: p. 107209.

14. Bruggink, S.C., et al., Cryotherapy with liquid nitrogen versus topical salicylic acid application for cutaneous warts in primary care: randomized controlled trial. Cmaj, 2010. 182(15): p. 162430.

15. Sterling, J.C., et al., British Association of Dermatologists' guidelines for the management of cutaneous warts 2014. Br J Dermatol, 2014. 171(4): p. 696-712.

16. Kwok, C.S., et al., Topical treatments for cutaneous warts. Cochrane Database Syst Rev, 2012. 2012(9): p. Cd001781.

17. Iranmanesh, B., et al., Laser therapy in cutaneous and genital warts: A review article. Dermatol Ther, 2021. 34(1): p. e14671.

18. Al-Hamamy, H.R., H.A. Salman, and N.A. Abdulsattar, Treatment of plane warts with a lowdose oral isotretinoin. ISRN Dermatol, 2012. 2012: p. 163929.

19. Hsu, V.M., et al., Efficacy of Nd:YAG laser therapy for the treatment of verrucae: a literature review. Lasers Med Sci, 2017. 32(5): p. 1207-1211.

20. Naz, R.K. and P. Dabir, Peptide vaccines against cancer, infectious diseases, and conception. Front Biosci, 2007. 12: p. 1833-44. 
21. Malonis, R.J., J.R. Lai, and O. Vergnolle, Peptide-Based Vaccines: Current Progress and Future Challenges. Chem Rev, 2020. 120(6): p. 3210-3229.

22. Liu, T.Y., et al., Advances in peptide-based human papillomavirus therapeutic vaccines. Curr Top Med Chem, 2012. 12(14): p. 1581-92.

23. Skwarczynski, M. and I. Toth, Peptide-based synthetic vaccines. Chem Sci, 2016. 7(2): p. 842854.

24. Soria-Guerra, R.E., et al., An overview of bioinformatics tools for epitope prediction: implications on vaccine development. J Biomed Inform, 2015. 53: p. 405-14.

25. Slota, M., et al., ELISpot for measuring human immune responses to vaccines. Expert Rev Vaccines, 2011. 10(3): p. 299-306.

26. Zhang, W., et al., ELISPOT assays provide reproducible results among different laboratories for $T$-cell immune monitoring--even in hands of ELISPOT-inexperienced investigators. $\mathrm{J}$ Immunotoxicol, 2009. 6(4): p. 227-34.

27. Weiskopf, D., et al., Comprehensive analysis of dengue virus-specific responses supports an HLA-linked protective role for CD8+T cells. Proc Natl Acad Sci U S A, 2013. 110(22): p. E2046-53.

28. Paul, S., et al., HLA class I alleles are associated with peptide-binding repertoires of different size, affinity, and immunogenicity. J Immunol, 2013. 191(12): p. 5831-9.

29. Sette, A., et al., The relationship between class I binding affinity and immunogenicity of potential cytotoxic T cell epitopes. J Immunol, 1994. 153(12): p. 5586-92.

30. Greenbaum, J., et al., Functional classification of class II human leukocyte antigen (HLA) molecules reveals seven different supertypes and a surprising degree of repertoire sharing across supertypes. Immunogenetics, 2011. 63(6): p. 325-35.

31. Andreatta, M. and M. Nielsen, Gapped sequence alignment using artificial neural networks: application to the MHC class I system. Bioinformatics, 2016. 32(4): p. 511-7.

32. Buus, S., et al., Sensitive quantitative predictions of peptide-MHC binding by a 'Query by Committee' artificial neural network approach. Tissue Antigens, 2003. 62(5): p. 378-84.

33. Lundegaard, C., et al., NetMHC-3.0: accurate web accessible predictions of human, mouse and monkey MHC class I affinities for peptides of length 8-11. Nucleic Acids Res, 2008. 36(Web Server issue): p. W509-12.

34. Lundegaard, C., O. Lund, and M. Nielsen, Accurate approximation method for prediction of class I MHC affinities for peptides of length 8, 10 and 11 using prediction tools trained on 9mers. Bioinformatics, 2008. 24(11): p. 1397-8.

35. Lundegaard, C., M. Nielsen, and O. Lund, The validity of predicted T-cell epitopes. Trends Biotechnol, 2006. 24(12): p. 537-8.

36. Nielsen, M., et al., Reliable prediction of T-cell epitopes using neural networks with novel sequence representations. Protein Sci, 2003. 12(5): p. 1007-17.

37. Hoof, I., et al., NetMHCpan, a method for MHC class I binding prediction beyond humans. Immunogenetics, 2009. 61(1): p. 1-13.

38. Jurtz, V., et al., NetMHCpan-4.0: Improved Peptide-MHC Class I Interaction Predictions Integrating Eluted Ligand and Peptide Binding Affinity Data. J Immunol, 2017. 199(9): p. 33603368 . 
39. Nielsen, M. and M. Andreatta, NetMHCpan-3.0; improved prediction of binding to MHC class I molecules integrating information from multiple receptor and peptide length datasets. Genome Med, 2016. 8(1): p. 33.

40. Nielsen, M., et al., NetMHCpan, a method for quantitative predictions of peptide binding to any HLA-A and -B locus protein of known sequence. PLoS One, 2007. 2(8): p. e796.

41. Reynisson, B., et al., NetMHCpan-4.1 and NetMHCIIpan-4.0: improved predictions of MHC antigen presentation by concurrent motif deconvolution and integration of MS MHC eluted ligand data. Nucleic Acids Res, 2020. 48(W1): p. W449-w454.

42. Zhang, H., O. Lund, and M. Nielsen, The PickPocket method for predicting binding specificities for receptors based on receptor pocket similarities: application to MHC-peptide binding. Bioinformatics, 2009. 25(10): p. 1293-9.

43. Jensen, K.K., et al., Improved methods for predicting peptide binding affinity to MHC class II molecules. Immunology, 2018. 154(3): p. 394-406.

44. Nielsen, M. and O. Lund, NN-align. An artificial neural network-based alignment algorithm for MHC class II peptide binding prediction. BMC Bioinformatics, 2009. 10: p. 296.

45. Nielsen, M., C. Lundegaard, and O. Lund, Prediction of MHC class II binding affinity using SMM-align, a novel stabilization matrix alignment method. BMC Bioinformatics, 2007. 8: p. 238.

46. Sidney, J., et al., Quantitative peptide binding motifs for 19 human and mouse MHC class I molecules derived using positional scanning combinatorial peptide libraries. Immunome Res, 2008. 4: p. 2.

47. Sturniolo, T., et al., Generation of tissue-specific and promiscuous HLA ligand databases using DNA microarrays and virtual HLA class II matrices. Nat Biotechnol, 1999. 17(6): p. 555-61.

48. Wang, P., et al., A systematic assessment of MHC class II peptide binding predictions and evaluation of a consensus approach. PLoS Comput Biol, 2008. 4(4): p. e1000048.

49. Wang, P., et al., Peptide binding predictions for HLA DR, DP and DQ molecules. BMC Bioinformatics, 2010. 11: p. 568.

50. Dhanda, S.K., P. Vir, and G.P. Raghava, Designing of interferon-gamma inducing MHC class-II binders. Biol Direct, 2013. 8: p. 30.

51. Bui, H.H., et al., Predicting population coverage of T-cell epitope-based diagnostics and vaccines. BMC Bioinformatics, 2006. 7: p. 153.

52. Valiyari, S., M. Salimi, and S. Bouzari, Novel fusion protein NGR-sIL-24 for targetedly suppressing cancer cell growth via apoptosis. Cell Biol Toxicol, 2020. 36(2): p. 179-193.

53. Wilkins, M.R., et al., Protein identification and analysis tools in the ExPASy server. Methods Mol Biol, 1999. 112: p. 531-52.

54. Agostini, F., et al., ccSOL omics: a webserver for solubility prediction of endogenous and heterologous expression in Escherichia coli. Bioinformatics, 2014. 30(20): p. 2975-7.

55. Dimitrov, I., et al., AllergenFP: allergenicity prediction by descriptor fingerprints. Bioinformatics, 2014. 30(6): p. 846-51.

56. Madeira, F., et al., The EMBL-EBI search and sequence analysis tools APIs in 2019. Nucleic Acids Res, 2019. 47(W1): p. W636-w641.

57. Lukács, A., et al., The quadrivalent HPV vaccine is protective against genital warts: a metaanalysis. BMC Public Health, 2020. 20(1): p. 691.

58. Yang, M.Y., et al., Quadrivalent human papilloma virus vaccine for the treatment of multiple warts: a retrospective analysis of 30 patients. J Dermatolog Treat, 2019. 30(4): p. 405-409. 
467 59. Lipke, M.M., An armamentarium of wart treatments. Clin Med Res, 2006. 4(4): p. 273-93.

468 60. Sanches, R.C.O., et al., Immunoinformatics Design of Multi-Epitope Peptide-Based Vaccine Against Schistosoma mansoni Using Transmembrane Proteins as a Target. Front Immunol, 2021. 12: p. 621706.

471 61. Schellenbacher, C., R.B.S. Roden, and R. Kirnbauer, Developments in L2-based human

472 papillomavirus (HPV) vaccines. Virus Res, 2017. 231: p. 166-175. vaccine in Escherichia coli. Can J Microbiol, 2017. 63(6): p. 493-501.

63. Mousavi, T., et al., Different types of adjuvants in prophylactic and therapeutic human papillomavirus vaccines in laboratory animals: a systematic review. Arch Virol, 2020. 165(2): p. 263-284. epitope vaccine. Infect Genet Evol, 2018. 58: p. 96-109. 
MHC-I \& MHC-II binding prediction

\section{Binding affinity cutoff}

\section{Overlapping analysis}

IFN- $\gamma$ inducer prediction (MHC-II)

Final epitope selection

Toxicity \& population coverage prediction 


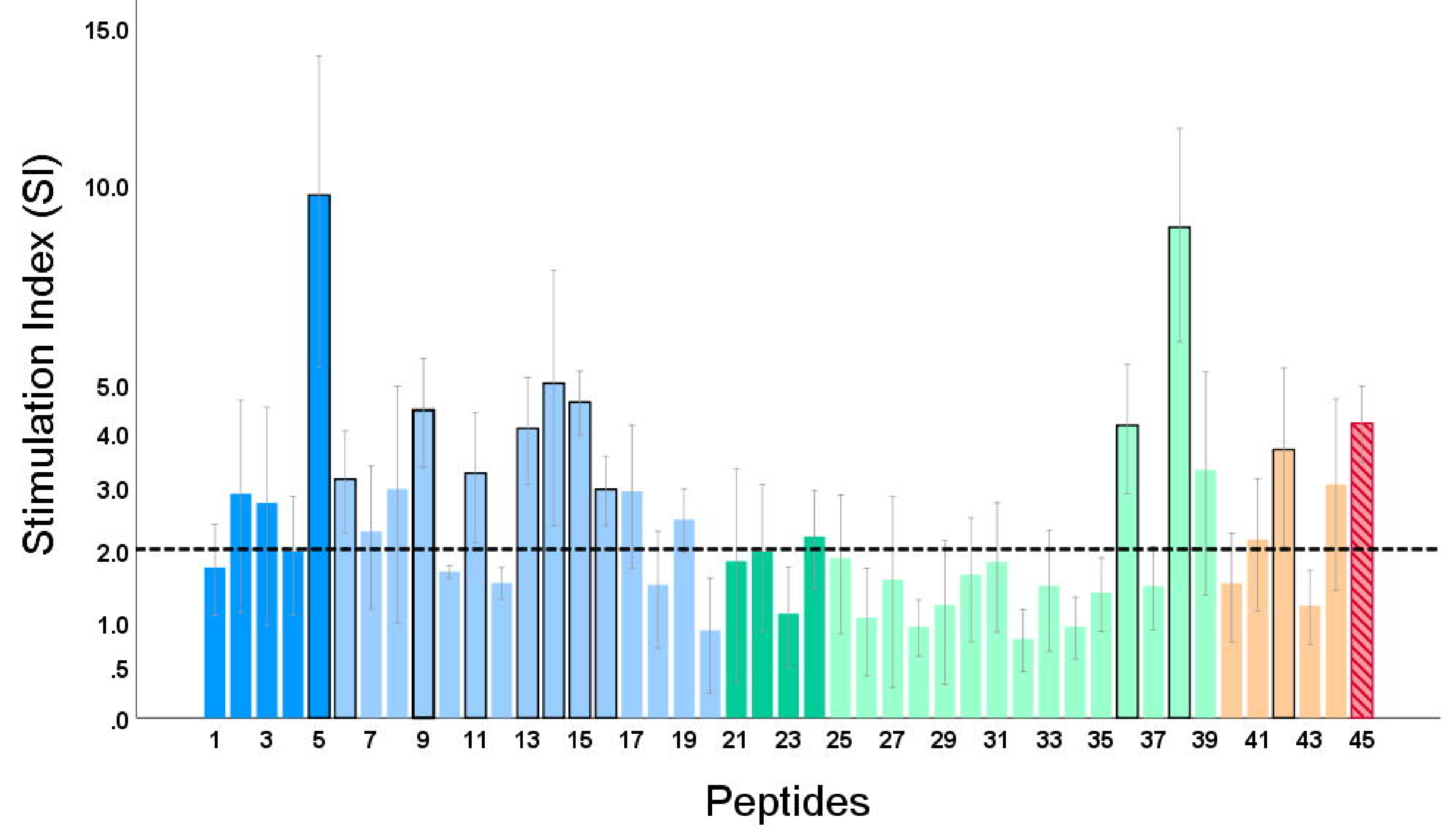


Unstimulated

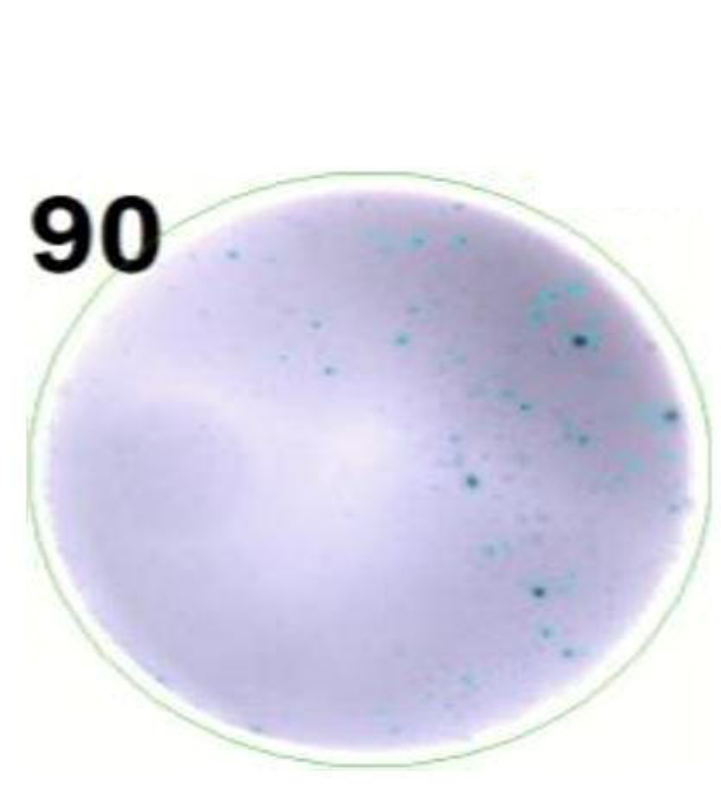

Stimulated with

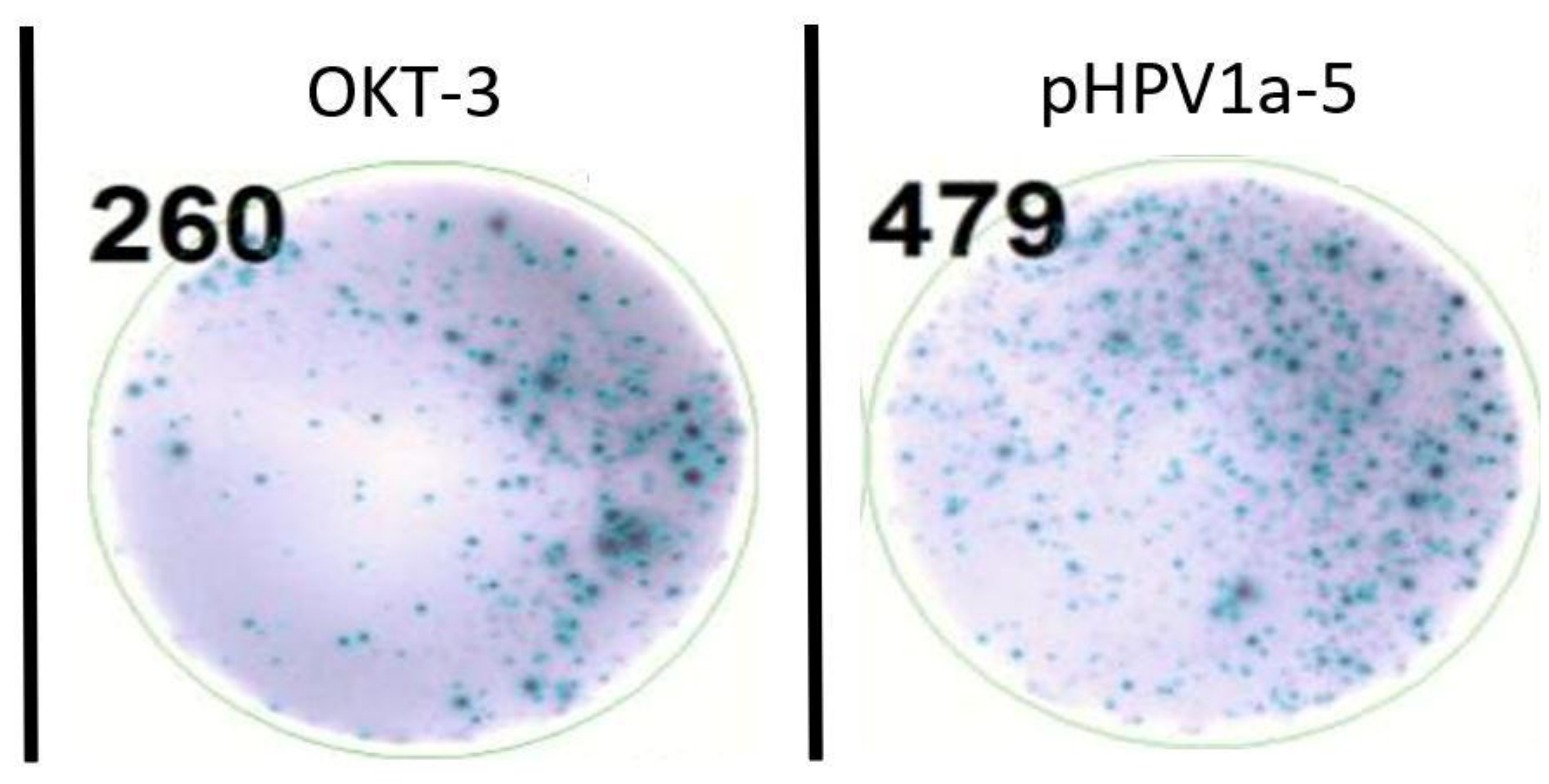




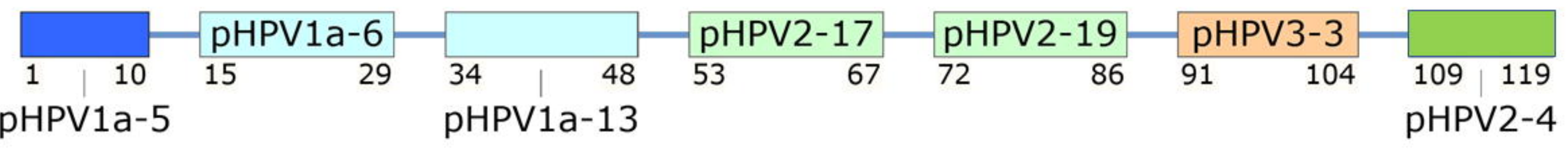

\begin{abstract}
KLTPENLAYIGGGSPNAFRVFRVRF ADPNGGGSYRFLGSSLAAKCPEQG GGSTQRLLWACVGVEVGRGGGSR TNVYYHGGSSRLLTGGGSGVEVGR GLPLGVGLGGGSFLLQRGAMPTV
\end{abstract}



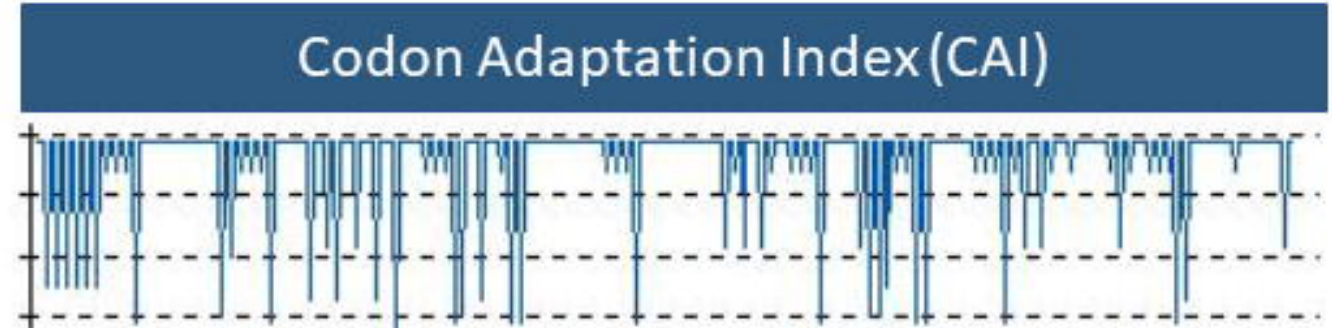

bioRxiv preprint doi: https://doi.org/10.1101/2021.09.24.461620; this version posted September 24, 2021. The copyright holder for this pre - (which was not certified by peer review)_is the_author/funder. All rights_reserved. No reuse allowed without permission.

a)

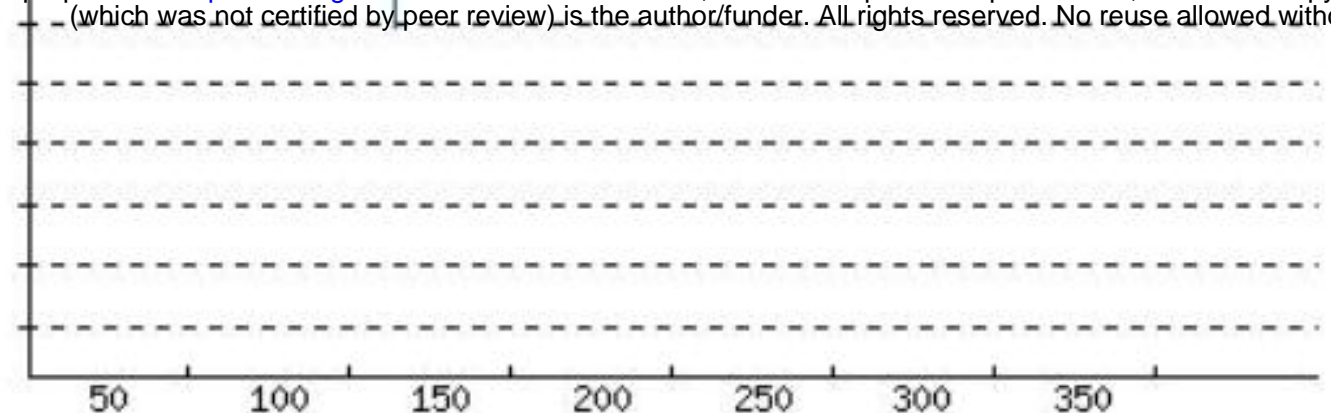

\section{CAl: 0.90}
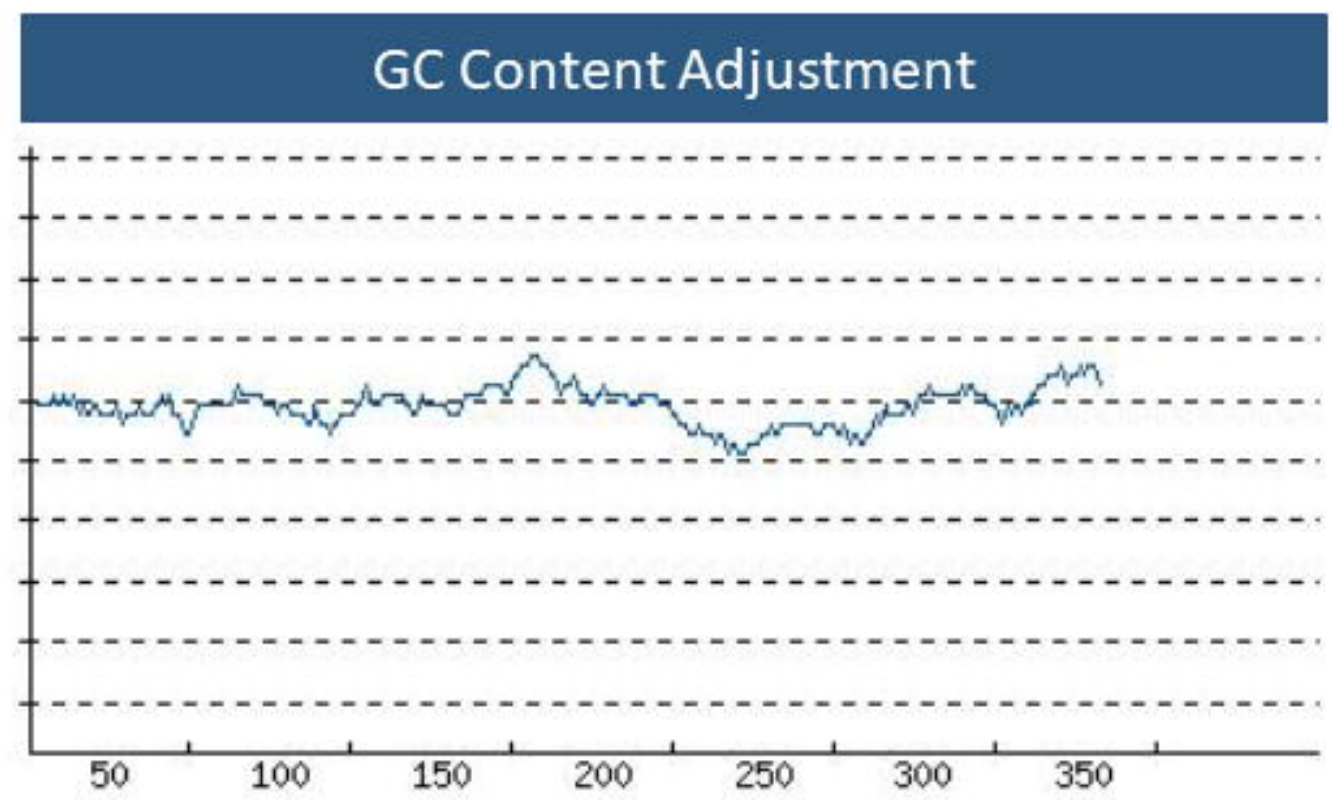

b) Average GC Content: $\mathbf{5 8 . 0 8 \%}$

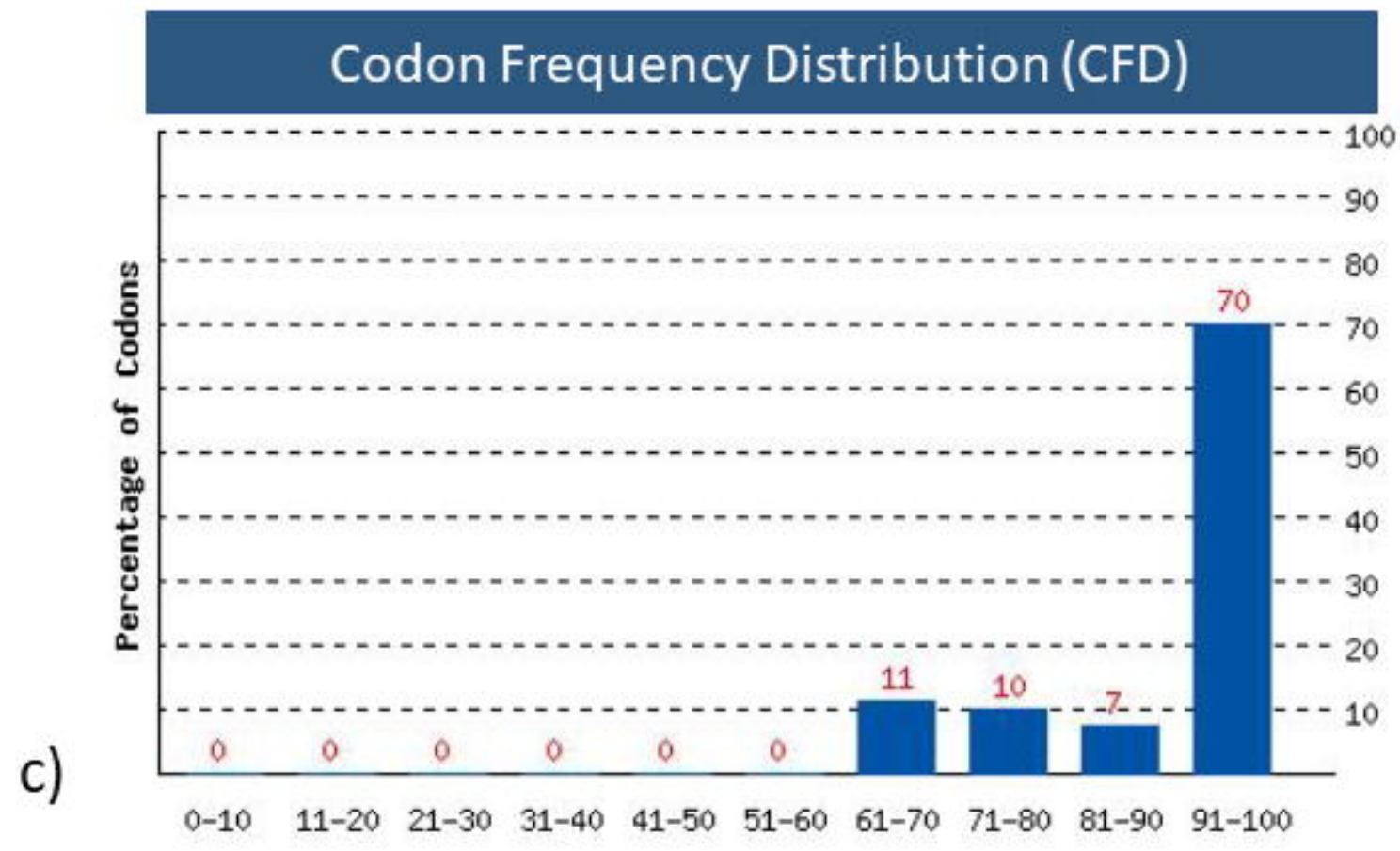

